\title{
NOTA SOBRE LA DISTRIBUCIÓN GEOGRÁFICA Y MORFOLOGÍA DE SISYRINCHIUM PLATYPHYLLUM (IRIDACEAE: SISYRINCHIEAE)
}

\author{
Daniela Martínez-JuÁrez, Noemí JimÉnez-Reyes y Aarón Rodríguez ${ }^{1}$
}

Universidad de Guadalajara, Centro Universitario de Ciencias Biológicas y Agropecuarias, Departamento de Botánica y Zoología, Instituto de Botánica, Apdo. postal 1-139, 45101 Zapopan, Jalisco, México.

${ }^{1}$ Autor para la correspondencia: rca08742@cucba.udg.mx

\begin{abstract}
RESUMEN
Se documentan seis nuevos registros de Sisyrinchium platyphyllum (Iridaceae) en el estado de Jalisco, México. Esta especie fue recolectada por primera vez en julio de 1889 en la barranca al norte de Guadalajara por el norteamericano Cyrus Guernsey Pringle. Desde entonces, no se habían recolectado otros ejemplares del taxon. Se incluyen las descripciones morfológica y palinológica completas así como imágenes y figuras de la especie.
\end{abstract}

Palabras clave: endemismo, florística, Iridaceae, Jalisco, México, Sisyrinchium.

\begin{abstract}
The presence of Sisyrinchium platyphyllum (Iridaceae) in six localities of the state of Jalisco, Mexico is documented. The species was first collected by Cyrus Guernsey Pringle on the southern rim of the Guadalajara canyon in 1889. Since then, its presence had not been documented again. Lastly, complete morphological and palynological descriptions and detailed figures are included.
\end{abstract}

Key words: endemism, floristic, Iridaceae, Jalisco, Mexico, Sisyrinchium.

\section{INTRODUCCIÓN}

El género Sisyrinchium L. (Iridaceae: Sisyrinchieae) agrupa entre 60 y 140 especies que se distribuyen en Norte, Centro y Sudamérica (Goldblatt et al., 1998; 
Goldblatt et al., 2008). En México se encuentran 42 de ellas, de las cuales 27 son endémicas (Espejo-Serna y López-Ferrari, 1996; Ceja-Romero et al., 1998, 2009; Espejo-Serna et al., 1998, 1999; Espejo-Serna et al., 2001; Espejo-Serna, 2012). Son plantas herbáceas, anuales o perennes que habitan en regiones templadas y tropicales. Poseen rizomas; raíces fibrosas y/o con engrosamientos tuberosos; hojas dísticas, lineares o ensiformes. Sus tallos florales producen hojas pero la longitud de la lámina disminuye hasta convertirse en brácteas. La inflorescencia es un ripidio que contiene dos a muchas flores pediceladas, actinomorfas y cubiertas por dos brácteas espatáceas. Los estambres se insertan en la base de los tépalos, los filamentos pueden estar libres o unidos formando un tubo. El fruto es una cápsula globosa, elipsoide u oblonga, pubescente o glabra, con semillas pequeñas y numerosas de color negro (Espejo-Serna y López-Ferrari, 1998; Goldblatt et al., 1998; Calderón de Rzedowski y Rzedowski, 2001; Espejo-Serna et al., 2010).

Sisyrinchium platyphyllum S. Watson es una especie endémica de Jalisco. En 1889, fue recolectada por primera vez por Cyrus Guernsey Pringle en una barranca cercana a Guadalajara. El primer viaje de Pringle a esta zona se realizó en 1888. Desde entonces y entusiasmado por la riqueza de la flora y la exuberancia de la vegetación de las barrancas del río Santiago, Pringle regresó una y otra vez a explorarlas. En total realizó 31 visitas a la zona entre 1888 y 1908, pasando la mayor parte de su tiempo en las cercanías de Guadalajara donde se concentró en la recolección de la flora de las barrancas (McVaugh, 1972). En este período, Pringle recolectó material de S. platyphyllum (Burns, 1936; McVaugh, 1989) y se estima que añadió al menos 600 nuevos registros a la flora de Jalisco, muchas de ellas novedades para la ciencia y descritas por Sereno Watson (Watson, 1891; McVaugh, 1972). Entre éstas se encuentra S. platyphyllum, cuya presencia en Jalisco no se había documentado desde entonces a pesar de que Ornelas (1987), Cházaro (1993), Guerrero (1994) y Harker (2002) realizaron exploraciones botánicas en la barranca del río Santiago y otros cañones aledaños.

La barranca del río Santiago, ubicada al norte de Guadalajara, se originó con el paso del río del mismo nombre y constituye el límite entre la Sierra Madre Occidental y el Eje Volcánico Transversal. El punto de inicio del cañón puede localizarse cerca de la población de Puente Grande, Jalisco. Su recorrido continúa hacia Nayarit en dirección noroeste y recibe varios nombres de acuerdo con las regiones que atraviesa: barranca de Colimilla, de Oblatos, de Huentitán, de Ibarra, de la Experiencia, de San Cristóbal y de Santa Rosa. Por su extensión, profundidad y condiciones climáticas, la barranca alberga una gran diversidad de especies vegetales, muchas de 
ellas endémicas. También es un corredor biológico entre los ecosistemas templados del centro de Jalisco y los ambientes tropicales de la costa.

El objetivo de este trabajo es dar a conocer la ubicación de nuevas localidades de Sisyrinchium platyphyllum en Jalisco, así como proporcionar las descripciones morfológica y palinológica completas de la especie.

Para realizar la descripción de los granos de polen, se tomaron muestras de los ejemplares botánicos recolectados por A. Rodríguez en 2007, mismos que están depositados en el herbario Luz María Villarreal de Puga del Instituto de Botánica de la Universidad de Guadalajara (IBUG). El estudio se llevó a cabo en el Laboratorio de Palinología del IBUG, utilizando la técnica de acetólisis de Erdtman (1943) y se hicieron preparaciones fijas utilizando gelatina glicerinada como medio de montaje. Las medidas que se incluyen en la descripción palinológica fueron obtenidas de 48 granos de polen seleccionados al azar (Cuadro 1). De 25 de ellos, en vista ecuatorial mayor, se midieron el eje polar $(\mathrm{P})$ y el eje ecuatorial mayor $(\mathrm{E})$. De otros 20, en vista polar, se midieron el eje ecuatorial mayor (E), el eje ecuatorial menor (e) y el largo y ancho del sulco. De tres más en vista ecuatorial menor se obtuvieron las medidas del eje polar (P) y el eje ecuatorial menor (e). Las observaciones y mediciones se realizaron en un microscopio de luz (ML) Carl Zeiss Standart K7 con cámara de circuito cerrado de TV. La terminología usada en la descripción palinológica es la propuesta por Erdtman (1972), Kremp (1965), Faegri e Iversen (1975) y Sáenz de Rivas (1978).

Cuadro 1. Resumen de las medidas de los granos de polen de Sisyrinchium platyphyllum. $\mathrm{E}=$ eje ecuatorial mayor. $\mathrm{e}=$ eje ecuatorial menor. $\mathrm{P}=$ eje polar. $\mathrm{L}=$ largo. $\mathrm{A}=$ ancho.

\begin{tabular}{|c|c|c|c|c|c|}
\hline Especímenes & $\begin{array}{l}\text { Núm. } \\
\text { de } \\
\text { granos }\end{array}$ & $\begin{array}{l}\text { Vista ecuatorial } \\
\text { mayor } \\
(\mathrm{Px} \mathrm{E})\end{array}$ & $\begin{array}{l}\text { Vista polar } \\
\text { (E x e) }\end{array}$ & $\begin{array}{l}\text { Vista ecuatorial } \\
\text { menor } \\
(\mathrm{Px} \mathrm{e})\end{array}$ & $\begin{array}{l}\text { Sulco } \\
(\mathrm{L} \times \mathrm{A})\end{array}$ \\
\hline $\begin{array}{l}\text { 1. Rodriguez } \\
\text { et al. } 5116\end{array}$ & 48 & $\begin{array}{c}23(26.5) 29.5 \mu \mathrm{m} \\
\mathrm{x} \\
45.5(48.5) 51.5 \mu \mathrm{m}\end{array}$ & $\begin{array}{c}44(48.6) 51 \mu \mathrm{m} \\
\mathrm{x} \\
25(29.2) 37 \mu \mathrm{m}\end{array}$ & $\begin{array}{c}28-31 \mu \mathrm{m} \\
\mathrm{x} \\
33-34 \mu \mathrm{m}\end{array}$ & $\begin{array}{c}40(45.2) 49 \mu \mathrm{m} \\
\mathrm{x} \\
1(5.6) 15 \mu \mathrm{m}\end{array}$ \\
\hline $\begin{array}{l}\text { A. Rodríguez } \\
\text { et al. } 5107\end{array}$ & 59 & $\begin{array}{c}25(29.6) 32 \mu \mathrm{m} \\
\mathrm{x} \\
42(47.7) 55 \mu \mathrm{m}\end{array}$ & $\begin{array}{c}44(46.7) 50 \mu \mathrm{m} \\
\mathrm{x} \\
27(32.3) 37 \mu \mathrm{m}\end{array}$ & $\begin{array}{c}30-35 \mu \mathrm{m} \\
\mathrm{x} \\
33-38 \mu \mathrm{m}\end{array}$ & $\begin{array}{c}37(43.6) 50 \mu \mathrm{m} \\
\mathrm{x} \\
0.5(8) 19 \mu \mathrm{m}\end{array}$ \\
\hline $\begin{array}{r}\text { A. Rodríguez } \\
\text { et al. } 5133\end{array}$ & 38 & $\begin{array}{c}16(23.8) 27 \mu \mathrm{m} \\
\mathrm{x} \\
39.5(45) 52.5 \mu \mathrm{m}\end{array}$ & $\begin{array}{c}43.5(48.7) 56 \mu \mathrm{m} \\
\mathrm{x} \\
24.5(28) 31 \mu \mathrm{m}\end{array}$ & $\begin{array}{c}22-24 \mu \mathrm{m} \\
\mathrm{x} \\
29.5-32 \mu \mathrm{m}\end{array}$ & $\begin{array}{c}38(44) 52 \mu \mathrm{m} \\
\mathrm{x} \\
0.3(2.3) 5 \mu \mathrm{m}\end{array}$ \\
\hline
\end{tabular}




\section{RESULTADOS}

Sisyrinchium platyphyllum S. Watson, Proc. Amer. Acad. Arts 26: 155-156. 1891. TIPO: Mexico, Jalisco, barranca near Guadalajara, C. G. Pringle 2876, (holotipo: GH, isotipos NY!, VT!). Figs. 1 y 2.

Hierbas perennes, glabras, glaucas, de $20-75 \mathrm{~cm}$ de alto; raíces fasciculadotuberosas, fusiformes, los engrosamientos alejados del tallo, de (5)7-11 cm de largo; hojas basales 2-9, envainantes, las vainas de color pardo hacia la base, de 9-13 cm de largo, las láminas ensiformes, falcadas, a veces rectas, agudas, de $15-32 \mathrm{~cm}$ de largo y de $0.5-1.5 \mathrm{~cm}$ de ancho, lisas, con el margen entero; vainas de las hojas viejas desintegrándose y formando un collar de fibras en la base de la planta; tallos florales glaucos, ramificándose hacia la porción apical, con 3-5 entrenudos, aplanados, alados, ciliados, terminando en la base de la inflorescencia, la porción inferior del tallo comprimida; brácteas caulinares envainantes, glaucas, falcadas, la del primer nudo de $10-20 \mathrm{~cm}$ de largo y de $0.5-1 \mathrm{~cm}$ de ancho, las superiores más pequeñas; pedúnculos de 3.0-12.5 $\mathrm{cm}$ de largo, aplanados y alados, de 3-4.5 $\mathrm{mm}$ de ancho en la base de la espata; la inflorescencia es un ripidio con 6-9 flores, cubierto por un par de brácteas espatáceas desiguales, de 1.5-2.8 cm de largo y de $0.5-1.2(1.8) \mathrm{cm}$ de ancho, que forman una estructura elíptico-lanceolada, la bráctea exterior más corta que la interior, fusionada en la base a lo largo de 2-4 mm, márgenes hialinos, aguda; brácteas florales hialinas, de $2.5 \mathrm{~cm}$ de largo y de $3 \mathrm{~mm}$ de ancho; pedicelos filiformes, de 1.6-2.4 cm de largo, erectos en la floración y la fructificación; perianto amarillo, flores de ca. $3.5 \mathrm{~cm}$ diámetro; tépalos obovados, atenuados, obtusos, de 1.5 $\mathrm{cm}$ de largo y de $0.6 \mathrm{~cm}$ de ancho; filamentos unidos en la base formando un tubo de $2 \mathrm{~mm}$ de alto, la parte libre de $5 \mathrm{~mm}$ de largo; anteras amarillas, de 6-8 $\mathrm{mm}$ de largo; ovario de $4 \mathrm{~mm}$ de largo y de $2 \mathrm{~mm}$ de ancho, glandular puberulento; cápsulas elipsoides, de 0.3-1.2 cm de largo y de 2-8 $\mathrm{mm}$ de ancho, glandular puberulentos; semillas numerosas, globosas, de $0.5 \mathrm{~mm}$ de diámetro, negras.

Distribución y hábitat: Sisyrinchium platyphyllum es endémico de Jalisco. Se conocía, hasta ahora, únicamente de tres números de colecta obtenidos en o cerca de la localidad tipo (C. G. Pringle 2876, 1889; C. G. Pringle 3847, 1891 y C. G. Pringle 11192, 1902). Material recolectado recientemente documenta su presencia en los municipios de San Cristóbal de la Barranca y Zapopan en la zona de la barranca del río Santiago y en los municipios de Guachinango, Jocotepec, Tapalpa y Tecolotlán 
Martínez-Juárez et al.: Distribución geográfica y morfología de Sisyrinchium platyphyllum
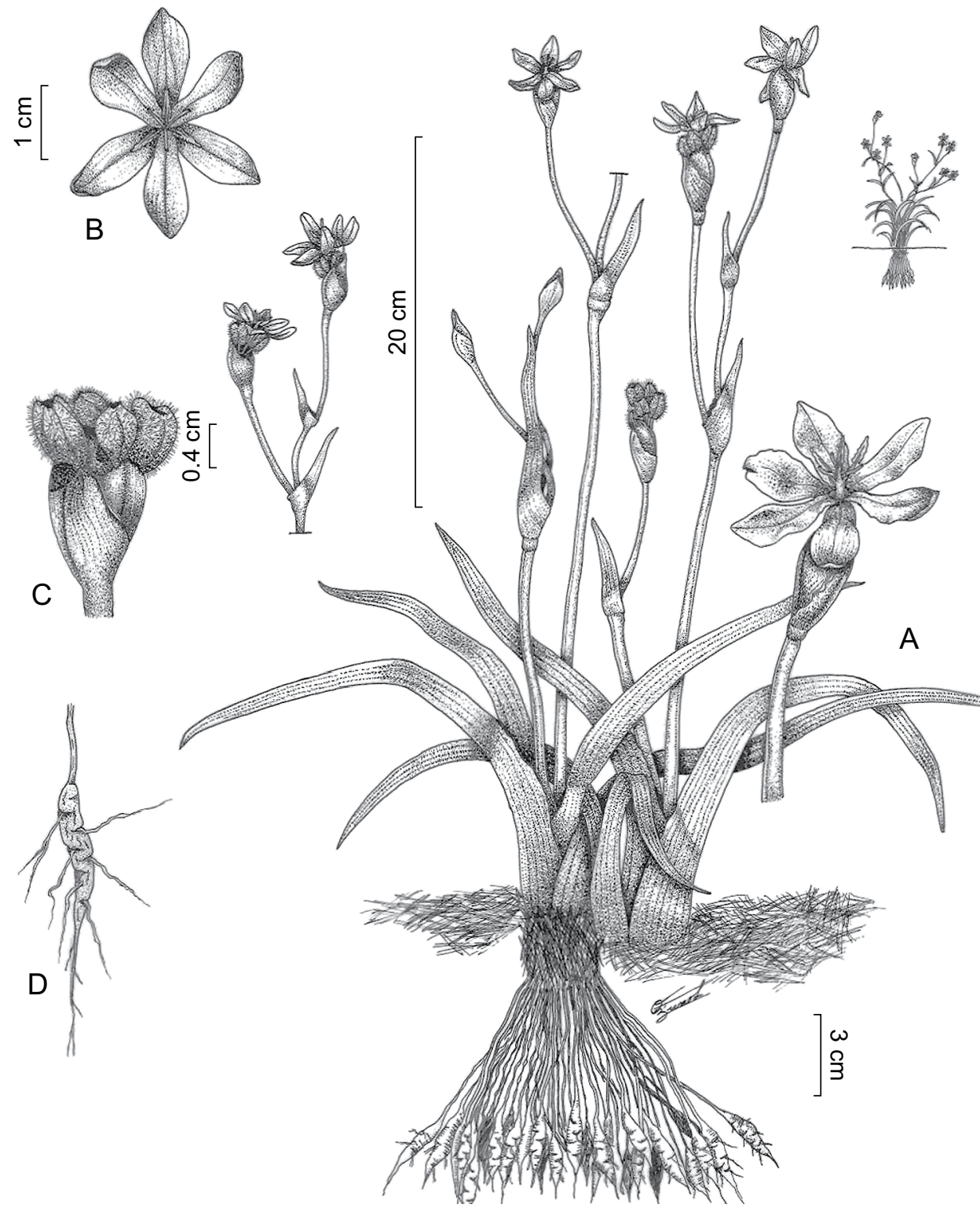

Fig. 1. Sisyrinchium platyphyllum. A. hábito; B. flor; C. fruto; D. raíz (Rodríguez et al. 5116, IBUG). 
(Fig. 3). El taxon crece en el pastizal derivado del bosque mixto de Quercus y Pi$n u s$, el encinar y el bosque tropical caducifolio sobre los taludes a lo largo de los
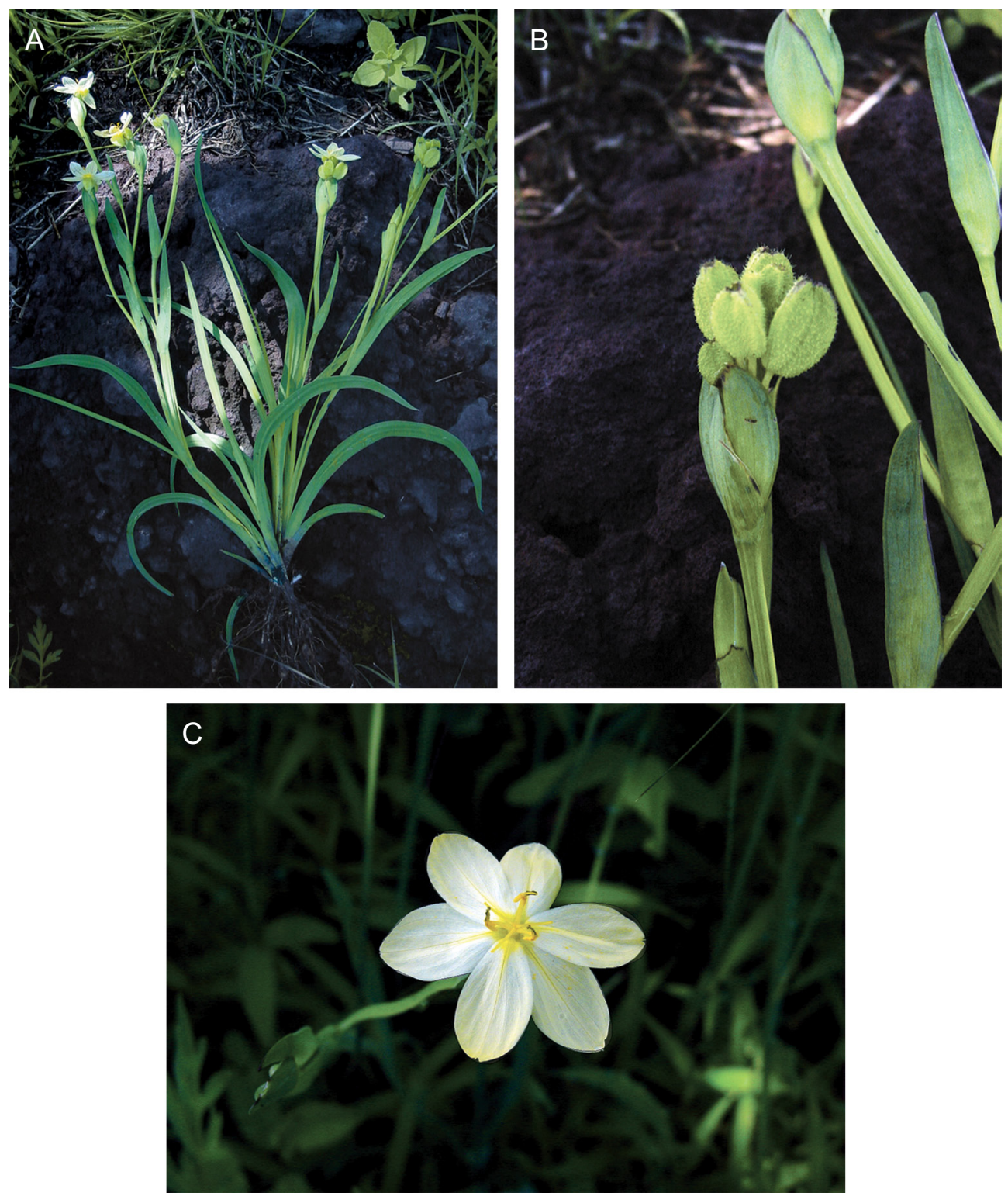

Fig. 2. Sisyrinchium platyphyllum. A. hábito; B. fruto; C. flor (A. Rodriguez et al. 5116, IBUG). 


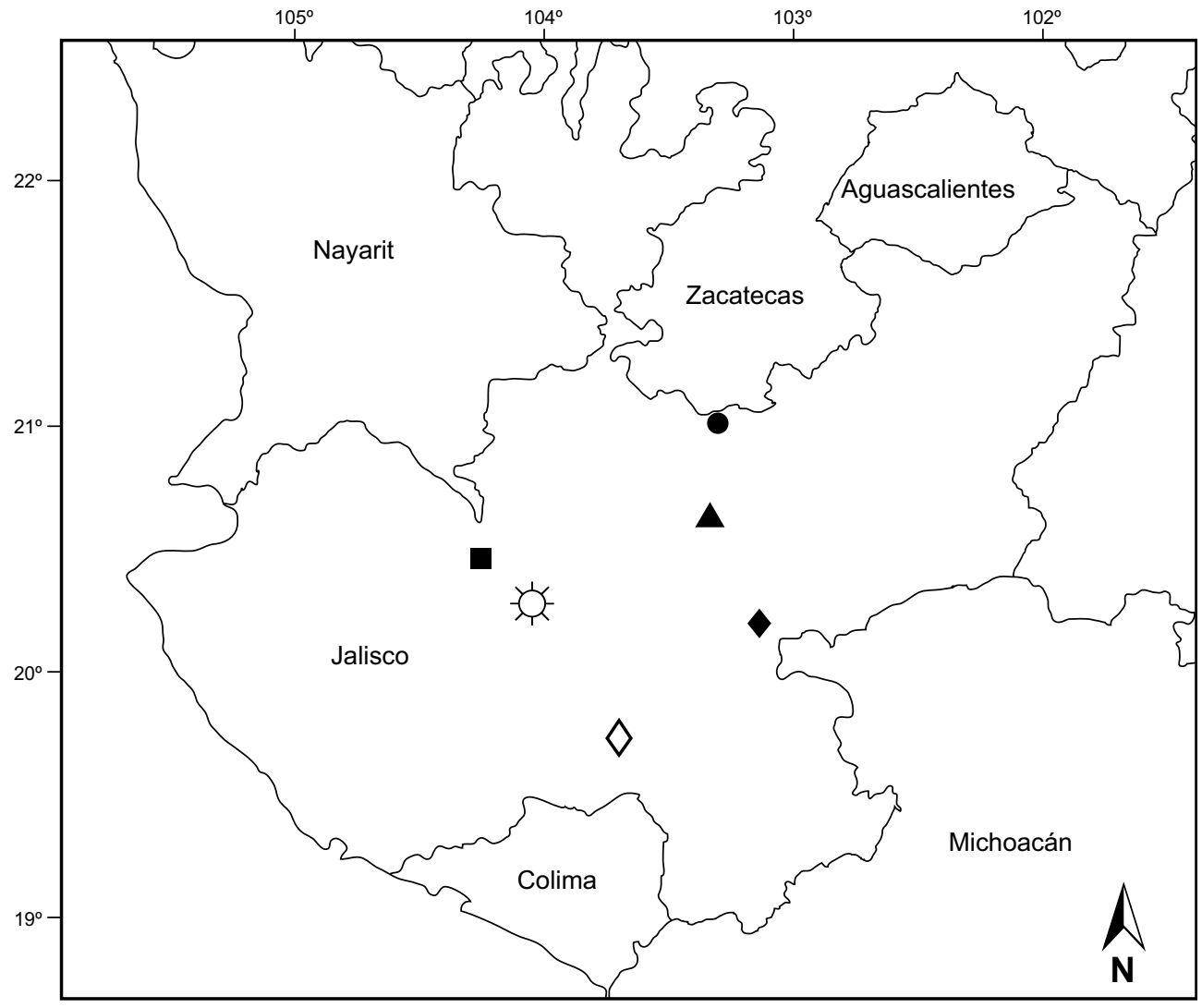

Fig. 3. Distribución geográfica de Sisyrinchium platyphyllum. (ロ) Guachinango; (»)

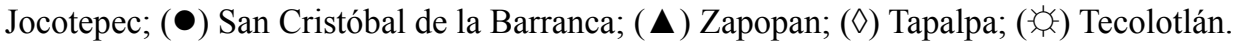

caminos entre los 1400 y $2000 \mathrm{~m}$. También se ha observado cerca de cultivos y en zonas con suelos muy húmedos. En los alrededores de San Cristóbal de la Barranca y de Zapopan, S. platyphyllum convive con Quercus resinosa Liebm., Lysiloma acapulcense (Kunth) Benth., Eysenhardtia polystachya (Ortega) Sarg., Calochortus purpureus (Kunth) Baker, C. hartwegii Benth., Polianthes zapopanensis E. Solano \& Ríos-Gómez, Manfreda scabra (Ortega) McVaugh, Tigridia mexicana Molseed ssp. passiflora Molseed, T. meleagris (Lindl.) G. Nicholson y Dahlia coccinea Cav. De forma similar, en la población de Guachinango crece junto a Quercus resinosa, Manfreda scabra, M. guttata (Jacobi \& C.D. Bouché) Rose, Polianthes longiflora Rose, P. platyphylla Rose, Tigridia mexicana Molseed ssp. lilacina Molseed, T. dugesii S. 
Watson, Calochortus purpureus, C. fuscus Schult. f., Milla biflora Cav., Bessera elegans Schult. f., Hymenocallis jaliscensis M.E. Jones y Echeandia pringlei Greenm.

Fenología: Sisyrinchium platyphyllum florece y fructifica de julio a septiembre.

Especímenes examinados: México. Jalisco: municipio de Guachinango, predio El Bajío, km 35 carretera Méx. 70 entre Ameca y Mascota, alt. 1540 m, 20³2'56" N, 104'18'8.3" W, 6.VIII.2007, A. Rodríguez et al. 5107 (CHAP, CHAPA, CIIDIR, EBUM, ENCB, GUADA, IBUG, IEB, MEXU, MICH, NY, OAX, TEX, WIS, XAL, ZEA); municipio de Jocotepec, paraje La Nariz, Cerro Viejo, frente al poblado de Zapotitán de Hidalgo, alt. 2000 m, 7.VII.1988, A. Machuca 6171 (IBUG); municipio de San Cristóbal de la Barranca, $500 \mathrm{~m}$ al sur del rancho El Malacate, casi en el límite estatal Jalisco-Zacatecas, alt. $1428 \mathrm{~m}, 21^{\circ} 08^{\prime} 37.3^{\prime \prime} \mathrm{N}, 103^{\circ} 25^{\prime} 32.5^{\prime \prime} \mathrm{W}, 6 . \mathrm{VII} .2003$, L. Ortiz-Catedral 231 (IBUG); municipio de San Cristóbal de la Barranca, km 51 carretera Zapopan-García de la Cadena, $500 \mathrm{~m}$ al sur de El Malacate, alt. $1428 \mathrm{~m}$, $21^{\circ} 08^{\prime} 37.3^{\prime \prime} \mathrm{N}, 103^{\circ} 25^{\prime} 32.5^{\prime \prime} \mathrm{W}, 9 . \mathrm{VIII} .2007$, A. Rodriguez et al. 5116 (CHAPA, CIIDIR, ENCB, IBUG, IEB, MEXU, NY, WIS, XAL, ZEA); municipio de Tapalpa, Cerro de Tlacozagua (on Sierra de Tapalpa) 3 km E of Tapalpa, alt. 2000 m, 19 57' N, 10345' W, 5.VIII.1960, H. Iltis et al. 784 (MICH, WIS); municipio de Tecolotlán, campamento La Ciénega, Sierra de Quila, alt. 1900 m, 30.VI.2000, M. Cházaro y J. Luna 8090 (IBUG); municipio de Zapopan, km 10.5 brecha Santa Lucía - La Coronilla, alt. $1643 \mathrm{~m}, 20^{\circ} 51.092^{\prime} \mathrm{N} ; 103^{\circ} 30.736^{\prime} \mathrm{W}, 16 . \mathrm{IX} .2006$, A. Rodríguez et al. 4922 (IBUG); municipio de Zapopan, km 6 camino entre Santa Lucía y La Coronilla, 1643 m, 2051.092' N, 103³0.736' W, 16.IX.2007, A. Rodríguez et al. 5133 (CHAPA, CIIDIR, ENCB, IBUG, IEB, MEXU, NY, WIS, XAL, ZEA).

Descripción palinológica (Fig. 4): grano heteropolar, bilateral, monosulcado, oblato, de 23(26.5)29.5 $\mu \mathrm{m} \times 45.5(48.5) 51.5 \mu \mathrm{m}$ en vista ecuatorial mayor. $\mathrm{P} / \mathrm{E}=0.55$. Vista ecuatorial menor de $28-31 \mu \times 33-34 \mu \mathrm{m} . \mathrm{P} / \mathrm{E}=0.86$, oblato esferoidal. Vista polar elíptica con los lados ligeramente convexos de 44(48.6)51 $\mu \mathrm{m}$ x 25(29.2)37 $\mu \mathrm{m}$ en sus ejes ecuatoriales. Exina semitectada, de 1.7(2)2.1 $\mu \mathrm{m}$ de grosor, sexina de $1(1.1) 1.2 \mu \mathrm{m}$ y nexina de $0.7(0.8) 0.9 \mu \mathrm{m}$ de espesor, microreticulada, heterobrocada; retículos poligonales; muros simplibaculados. Sulco de 40(45.2)49 $\mu \mathrm{m}$ de largo y 1(5.6)15 $\mu \mathrm{m}$ de ancho. La apertura monosulcada y la exina reticulada de Sisyrinchium platyphyllum son características que comparten las especies del género (González, 1969; Heusser, 1971; Erdtman, 1972; Markgraf y D’Antoni 1978; Zavada, 1983). 

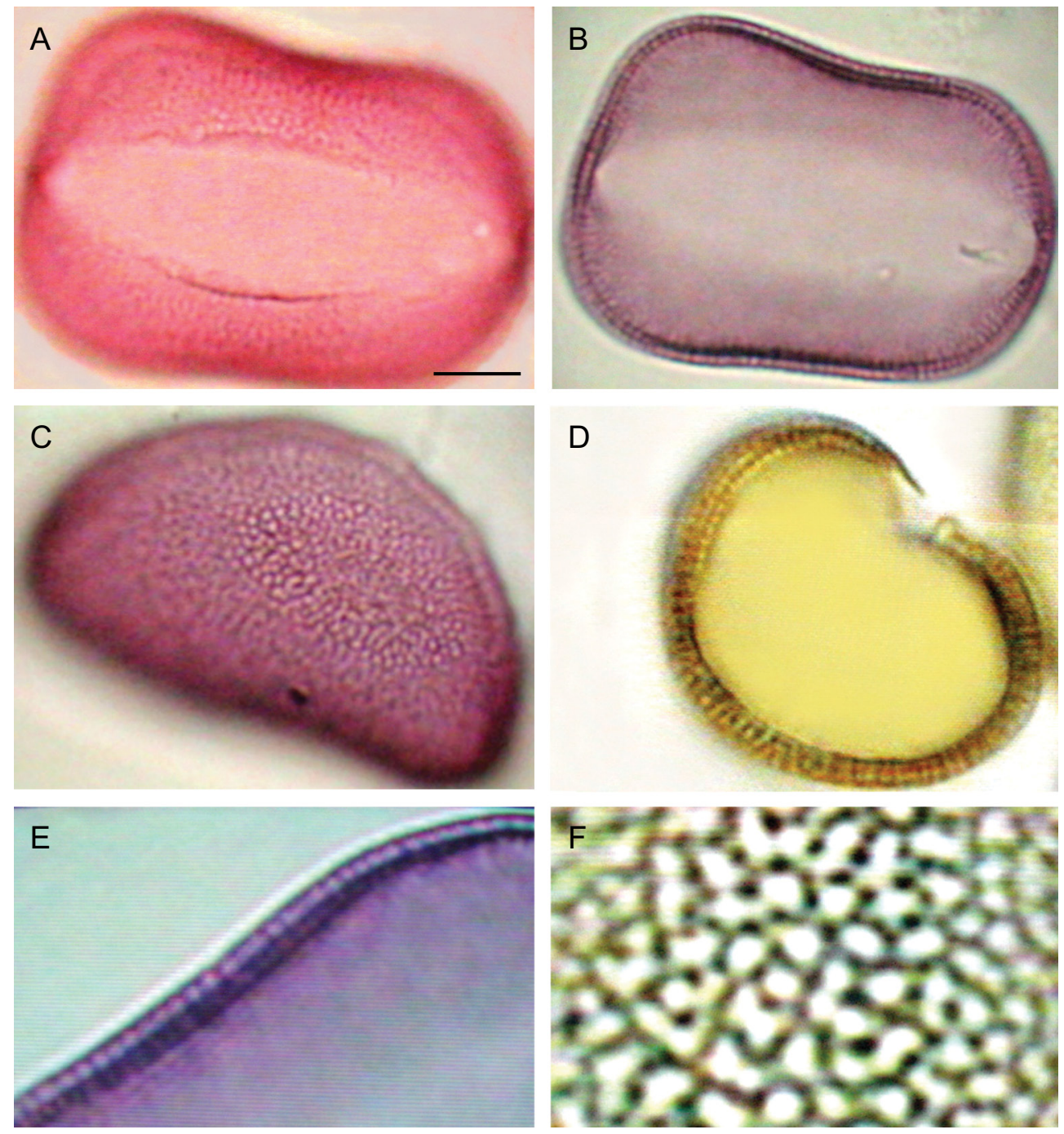

Fig. 4. Granos de polen de Sisyrinchium platyphyllum. A. vista polar, se muestra el sulco amplio; B. vista polar, corte óptico a nivel de la exina; C. vista ecuatorial mayor; D. vista ecuatorial menor, corte óptico; E. acercamiento de corte óptico a nivel de la exina; F. detalle de la superficie, se observa microretículo con muros simplibaculados. Escala $=8 \mu$ (en A-D), $4 \mu$ (en E) y $2.5 \mu$ (en F).

Especímenes de referencia: A. Rodríguez et al. 5116 (IBUG), A. Rodríguez et al. 5107 (IBUG) y A. Rodríguez et al. 5133 (IBUG) (Cuadro 1). 


\section{DISCUSIÓN}

Sisyrinchium platyphyllum incluye plantas de 20 a $75 \mathrm{~cm}$ de alto. Se caracteriza por tener hojas falcadas y glaucas, cápsulas glandular-puberulentas y los engrosamientos de la raíz alejados del tallo. Sus hojas falcadas lo hacen parecido a ejemplares robustos de $S$. convolutum pero este último tiene el ovario y el fruto glabros.

Otras especies morfológicamente similares a Sisyrinchium platyphyllum son S. arizonicum y S. macrophyllum. En estos taxones, el ovario y el fruto son pubescentes. La diferencia entre ellos está en las características de las hojas y de las raíces. En S. platyphyllum, las hojas son ensiformes y falcadas, contrastando con las hojas lineares y erectas de $S$. arizonicum y $S$. macrophyllum. Por su parte, S. arizonicum y $S$. macrophyllum tienen raíces cilíndricas y poco engrosadas, en contraste con $S$. platyphyllum que tiene raíces tuberosas y fusiformes.

Por su tamaño, el color amarillo de sus tépalos y el número de flores abiertas el mismo día, Sisyrinchium platyphyllum tiene un gran potencial como planta ornamental. Con base en el color y la estructura de sus flores, muchas iridáceas mexicanas han sido sugeridas como plantas ornamentales (Rodríguez y Ortiz-Catedral, 2003; Espejo-Serna y López-Ferrari, 2003).

Sisyrinchium platyphyllum se conocía, hasta ahora, únicamente de la localidad tipo y zonas aledañas, citadas por Pringle como "barranca near Guadalajara" y por Watson (1891) como "grassy bluffs of barranca near Guadalajara". En 1886, Edward Palmer colectó en las barrancas de Guadalajara (Watson, 1887). Las localidades fueron Río Blanco, San Esteban, Paso de Ibarra, La Barranca y 10 millas al norte de Guadalajara. Palmer atrajo la atención de Pringle sobre estos lugares, por lo que es posible que ésta sea la zona donde Pringle recolectó a $S$. platyphyllum en tres ocasiones. Los resultados de este trabajo resaltan la importancia de realizar exploraciones botánicas extensivas e intensivas. Si bien, la especie en cuestión habita una zona muy explorada botánicamente, hasta ahora había pasado desapercibida. $\mathrm{Su}$ distribución geográfica es menos restringida de lo que se había registrado y se amplía ahora con la existencia de seis nuevas localidades en Jalisco.

\section{AGRADECIMIENTOS}

Agradecemos a Osvaldo Zuno la elaboración de la figura 1. De igual forma, estamos agradecidos con Mollie Harker, Jorge Pérez de la Rosa, Raymundo Ramírez Delgadillo y Jacqueline Reynoso Dueñas por su apoyo durante la elaboración de este artículo. 


\section{LITERATURA CITADA}

Burns, D. H. 1936. Life and work of Cyrus Guernsey Pringle. University of Vermont. Burlington, USA. $756 \mathrm{pp}$.

Calderón de Rzedowski, G. y J. Rzedowski. 2001. Flora fanerogámica del Valle de México. 2a. ed. Instituto de Ecología, A. C. y Comisión Nacional para el Conocimiento y Uso de la Biodiversidad. Pátzcuaro, México. 1406 pp.

Ceja-Romero, J., A. Espejo-Serna y A. R. López-Ferrari. 1998. Sisyrinchium arguellesiae (Iridaceae: Sisyrinchieae) una nueva especie del estado de Querétaro. Acta Bot. Mex. 44: 87-91.

Ceja-Romero, J., A. Espejo-Serna y A. R. López-Ferrari. 2009. Una nueva especie de Sisyrinchium (Iridaceae) del estado de Guanajuato, México. Acta Bot. Mex. 87: 83-90.

Cházaro, M. 1993. Investigación preliminar sobre el estado de conservación de la comunidad biótica (flora y fauna) en la barranca de Huentitán, Jalisco. Tiempos de Ciencia 31: 28-40.

Erdtman, G. 1943. An introduction to pollen analysis. The Ronald Press Co. New York, USA. 239 pp.

Erdtman, G. 1972. Pollen morphology and plant taxonomy. Angiosperms (an introduction to palynology I). (Corrected reprint of the edition 1952 with a new addendum). Hafner Publishing. New York, USA. 553 pp.

Espejo-Serna, A. 2012. El endemismo en las Liliopsida mexicanas. Acta Bot. Mex. 100: 195-257.

Espejo-Serna, A. y A. R. López-Ferrari. 1996. Comentarios florísticos-ecológicos sobre las iridáceas mexicanas. Acta Bot. Mex. 34: 25-47.

Espejo-Serna, A. y A. R. López-Ferrari. 1998. Iridaceae. Flora de Veracruz 105: 1-58.

Espejo-Serna, A. y A. R. López-Ferrari. 2003. Las monocotiledóneas (Liliopsida) mexicanas con potencial ornamental. In: Mejía-Muñoz, J. M. y A. Espinosa-Flores (compiladores). Plantas nativas de México con potencial ornamental: análisis y perspectivas. Universidad Autónoma Chapingo. Texcoco, México. pp. 20-30.

Espejo-Serna, A., A. R. López-Ferrari y J. Ceja-Romero. 1998. Una nueva especie gipsófila de Sisyrinchium (Iridaceae: Sisyrinchieae) de México. Acta Bot. Mex. 45: 43-47.

Espejo-Serna, A., A. R. López-Ferrari y J. Ceja-Romero. 1999. Una nueva especie de Sisyrinchium (Iridaceae: Sisyrinchieae) del estado de Durango, México. Acta Bot. Mex. 49: 19-22.

Espejo-Serna, A., A. R. López-Ferrari y J. Ceja-Romero. 2010. Iridaceae. Flora del Bajío y de Regiones Adyacentes 166: 1-81.

Espejo-Serna, A., A. R. López-Ferrari, J. Ceja-Romero y A. Cholewa. 2001. Two new species of Mexican Iridaceae. Acta Bot. Mex. 55: 21-27.

Faegri, K. y J. Iversen. 1975. Textbook of pollen analysis. Third revised edition. Munksgaard. Copenhagen, Denmark. 295 pp.

Goldblatt, P., A. Rodríguez, M. P. Powell, T. J. Davies, J. C. Manning, M. van der Bank y V. Savolainen. 2008. Iridaceae "Out of Australasia"? Phylogeny, biogeography, and divergence time based on plastid DNA sequences. Syst. Bot. 33: 495-508. 
Goldblatt, P., J. C. Manning y P. Rudall. 1998. Iridaceae. In: Kubitzki, K. (ed.). The families and genera of vascular plants III: flowering plants, monocotyledons, Lilianae (except Orchidaceae). Springer. Berlín, Germany. pp. 295-335.

González, L. 1969. Morfología polínica: la flora del Valle del Mezquital, Hidalgo. Paleoecología 3. Instituto Nacional de Antropología e Historia. México, D.F., México. 187 pp.

Guerrero, S. 1994. Estudio florístico preliminar de las barrancas aledañas a la zona metropolitana de Guadalajara, Jalisco. Tesis de licenciatura. Universidad de Guadalajara. Guadalajara, México. 76 pp.

Harker, M. F. 2002. La familia Compositae (Asteraceae) en la Barranca del Río Santiago, municipio de Guadalajara, Jalisco, México: un modelo para la conservación. Tesis de Maestría. Universidad de Guadalajara. Guadalajara, México. 167 pp.

Heusser, C. J. 1971. Pollen and spores of Chile. The University of Arizona Press. Tucson, USA. 167 pp.

Kremp, G. O. 1965. Morphologic encyclopedia of palynology. The University of Arizona Press. Tucson, USA. 263 pp.

Markgraf, V. y H. L. D’Antoni. 1978. Pollen flora Argentina. Modern spore and pollen types of Pteridophyta, Gymnospermae \& Angiospermae. The University of Arizona Press. Tucson, USA. 208 pp.

McVaugh, R. 1972. Botanical exploration in Nueva Galicia, Mexico from 1970 to the present time. Contr. Univ. Mich. Herb. 9: 205-522.

McVaugh, R. 1989. Bromeliaceae to Dioscoreaceae. Flora Novo-Galiciana 15: 294-347.

Ornelas, R. 1987. Guía de la excursión a San Cristóbal de la Barranca. In: Zamudio-Ruiz, S., F. Guevara-Fefer y J. A. Pérez de la Rosa (coordinadores). Guías de excursiones botánicas en México, VIII. Sociedad Botánica de México. Guadalajara, México. pp. $19-48$.

Rodríguez, A. y L. Ortiz-Catedral. 2003. Algunas especies de plantas nativas con potencial ornamental del occidente de México. In: Carvajal, S. (ed.). Avances de la investigación científica en el CUCBA. Coordinación de Investigación, Centro Universitario de Ciencias Biológicas y Agropecuarias, Universidad de Guadalajara. Guadalajara, México. pp. 263-268.

Sáenz de Rivas, C. 1978. Polen y esporas. H. Blume. Madrid, España. 219 pp.

Watson, S. 1887. List of plants collected by Dr. Edward Palmer in the state of Jalisco, Mexico, in 1886. Proc. Amer. Acad. Arts 22: 396-465.

Watson, S. 1891. Descriptions of new Mexican species, collected chiefly by Mr. C. G. Pringle in 1889 and 1890. Proc. Amer. Acad. Arts 26: 131-158.

Zavada, M. S. 1983. Comparative morphology of monocot pollen and evolutionary trends of apertures and wall structure. Bot. Rev. 49: 331-379. 